\title{
Blinatumomab in pediatric patients with relapsed/ refractory acute lymphoblastic leukemia: results of the RIALTO trial, an expanded access study
}

\author{
Franco Locatelli ${ }^{1}$, Gerhard Zugmaier $\mathbb{1}^{2}$, Noemi Mergen², Peter Bader ${ }^{3}$, Sima Jeha ${ }^{4}$, Paul-Gerhardt Schlegel ${ }^{5}$, \\ Jean-Pierre Bourquin ${ }^{6}$, Rupert Handgretinger ${ }^{7}$, Benoit Brethon ${ }^{8}$, Claudia Rossig ${ }^{9}$ and Christiane Chen-Santel ${ }^{10}$
}

Dear Editor,

Although most children with B-cell precursor acute lymphoblastic leukemia (BCP-ALL) achieve first remission with conventional, risk-adapted protocols, relapse still occurs in $15-20 \%$ of patients ${ }^{1}$. Current salvage treatments are associated with acute and long-term toxicities, which may cause long-term sequelae and treatment-related (TR) death ${ }^{2}$. As minimal residual disease (MRD) is a strong predictor of relapse in BCP-ALL ${ }^{3}$, new treatments associated with reduced toxicity and high rates of MRD clearance are needed to improve outcomes for children with relapsed/refractory $(r / r)$ BCP-ALL.

Blinatumomab is a bispecific CD19-directed CD3 T-cell engager (BiTE) molecule shown to improve overall survival (OS) as compared with standard chemotherapy in adult patients with $\mathrm{r} / \mathrm{r}$ BCP-ALL, and to induce high rates of complete remission (CR) and complete MRD response $^{4-6}$. An international phase $1 / 2$ study established the recommended dose of blinatumomab in children and adolescents with $\mathrm{r} / \mathrm{r}$ BCP-ALL, and demonstrated its antileukemic activity across age and risk groups ${ }^{7}$.

We report on the safety and efficacy of blinatumomab in an open-label, single-arm, expanded access international study of pediatric patients with CD19-positive $\mathrm{r} / \mathrm{r}$ BCPALL (RIALTO trial, NCT02187354). The protocol was approved by the Institutional Review Board/Independent Ethics Committee at each site; informed consent was obtained from patients' legal guardians. Enrolled patients were aged $>28$ days to $<18$ years, with CD19-positive

\footnotetext{
Correspondence: Gerhard Zugmaier (gerhardz@amgen.com)

'Department of Hematology and Oncology, IRCCS Bambino Gesù Children's

Hospital, Rome, Sapienza, University of Rome, Rome, Italy

${ }^{2}$ Amgen Research (Munich) GmbH, Munich, Germany

Full list of author information is available at the end of the article
}

BCP-ALL in second or later relapse, any relapse after allogeneic hematopoietic stem cell transplantation (alloHSCT), or refractory to other treatments. All patients had $\geq 5 \%$ blasts or $<5 \%$ blasts but with MRD level $\geq 10^{-3}$, and adequate liver function at screening. Blinatumomab $\left(5-15 \mu \mathrm{g} / \mathrm{m}^{2}\right.$ per day) was administered as a 6-week induction cycle, comprising continuous infusion for 4 weeks, followed by a 2 -week treatment-free period. Up to two induction cycles could be performed. Those achieving CR could then receive up to three additional consolidation cycles for a total of five cycles. Additional eligibility criteria, dosing information, and dose interruption or discontinuation criteria are included in Supplementary Material.

The primary endpoint was incidence of treatmentemergent (TE) and TR adverse events (AEs). Secondary endpoints included morphologic $\mathrm{CR}(<5 \%$ blasts $)$ and MRD response $\left(<10^{-4}\right.$ leukemic blasts by flow cytometry) in the first two cycles, relapse-free survival (RFS), OS, alloHSCT rate after blinatumomab treatment, and 100day mortality after alloHSCT. Bone marrow aspirate or biopsy was performed at screening, after blinatumomab infusion on day 29 of each treatment cycle, and 6-monthly during follow-up, until 18 months after the first blinatumomab dose. Statistical analyses are described in Supplementary Material.

The study began in 2014. Data cut-off for the current analysis was July 19, 2019. Baseline demographics and clinical characteristics for all patients $(n=110$; full analysis set) are shown in Supplementary Table 1. Eleven percent $(n=12)$ had $<5 \%$ blasts (with $\mathrm{MRD} \geq 10^{-3}$ ) at baseline. Thirty-two patients remained on treatment at the cut-off date (Supplementary Fig. 1). 


A
All patients with $\geq 5 \%$ blasts at baseline
Baseline blast category
$\geq 5-50 \%$
$\geq 50 \%$
Prior relapses
0
1
$\geq 2$
Prior HSCT
No
Yes

B

All patients with CR in first 2 cycles
Baseline blast category
$\quad<5 \%$
$\geq 5-50 \%$
$\geq 50 \%$
Prior relapses
0
1
$\geq 2$
Prior HSCT
$\quad$ No
Yes

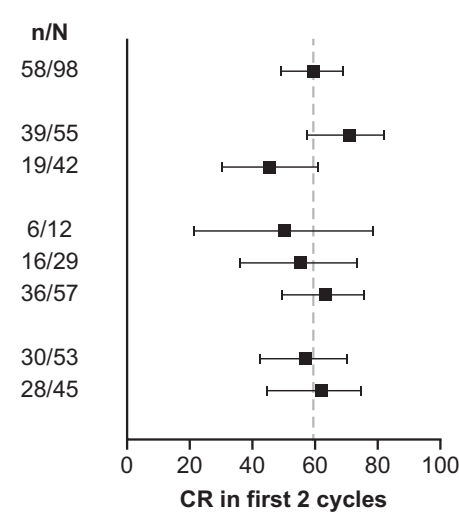

CR\% $(95 \% \mathrm{Cl})$

$59.2(48.8-69.0)$

$70.9(57.1-82.4)$

$45.2(29.8-61.3)$

$50.0(21.1-78.9)$

$55.2(35.7-73.6)$

$63.2(49.3-75.6)$

$56.6(42.3-70.2)$

$62.2(46.5-76.2)$

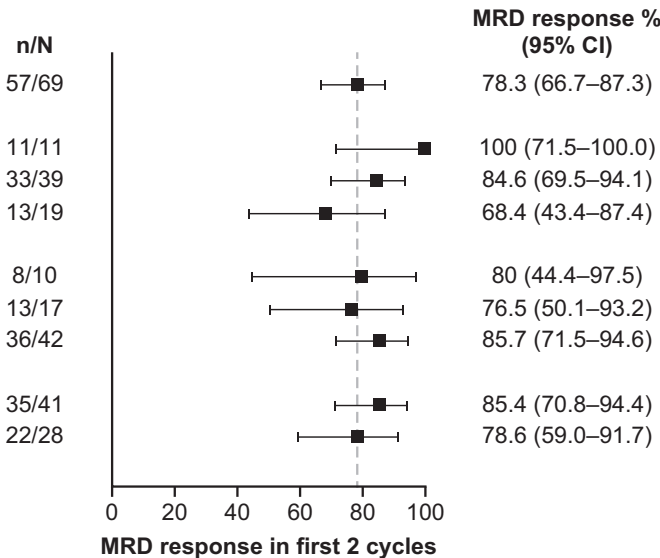

Fig. 1 Response rates within the first 2 cycles of blinatumomab treatment, by subgroup. a Complete remission (CR) rates in patients with $\geq 5 \%$ blasts at baseline. $\mathbf{b}$ Minimal residual disease (MRD) response rates.

Almost all patients (99\%) experienced TEAEs (Supplementary Table 2); AEs of $\geq$ grade 3 were experienced by $65 \%$ of patients. TRAEs were reported in $74 \%$ of patients; $26 \%$ were $\geq$ grade 3 (Supplementary Table 2). TRAEs leading to blinatumomab discontinuation or temporary interruption occurred in $4 \%$ and $16 \%$ of patients, respectively. There were nine fatal AEs; none were attributed to blinatumomab. Forty-two percent of patients experienced neurologic AEs, most commonly headache (25\%). Six patients (5.5\%) experienced grade 3 neurologic events: two patients experienced headache, and one patient each had depressed level of consciousness, seizure, trigeminal nerve disorder, and agitation. AEs were managed per protocol (see "Treatments" in Supplementary Material). There were no grade 4 or 5 neurologic AEs. Two patients (1.8\%) experienced grade $\geq 3$ cytokine release syndrome (CRS), and one (1\%) had grade 4 CRS.

Among patients with $\geq 5 \%$ blasts at baseline $(n=98), 58$ patients (59\%) achieved CR within the first two blinatumomab cycles. Of these, $39(67 \%)$ also achieved full hematologic recovery and $46(79 \%)$ achieved MRD response (Supplementary Table 3). Details of patients with nonevaluable or unavailable response data are given in the Supplementary Material. Of the twelve patients enrolled with $<5 \%$ blasts, 11 (92\%) achieved MRD response during the first two blinatumomab cycles including three with full recovery of peripheral blood counts, while response data were unavailable for one patient.

Overall, the response rate was higher among patients with lower baseline tumor burden (Fig. 1). Four of five patients with prior blinatumomab exposure achieved $\mathrm{CR}$ with full recovery of peripheral blood counts, and three achieved MRD response in the first two cycles. Two patients had $t$ $(17 ; 19)$ rearrangement and both achieved CR with MRD response in the first two treatment cycles; both completed the study in remission and were alive at last follow-up. Three of the four patients with constitutional trisomy 21 achieved CR with MRD response, two completed the study in remission and were still alive at last follow-up.

Of 110 patients in the study, 69 patients had CR as best response in the first two cycles; of these, 45 (65\%) proceeded to alloHSCT. Simon-Makuch 42-day landmark analyses showed a trend toward improved OS and RFS for 


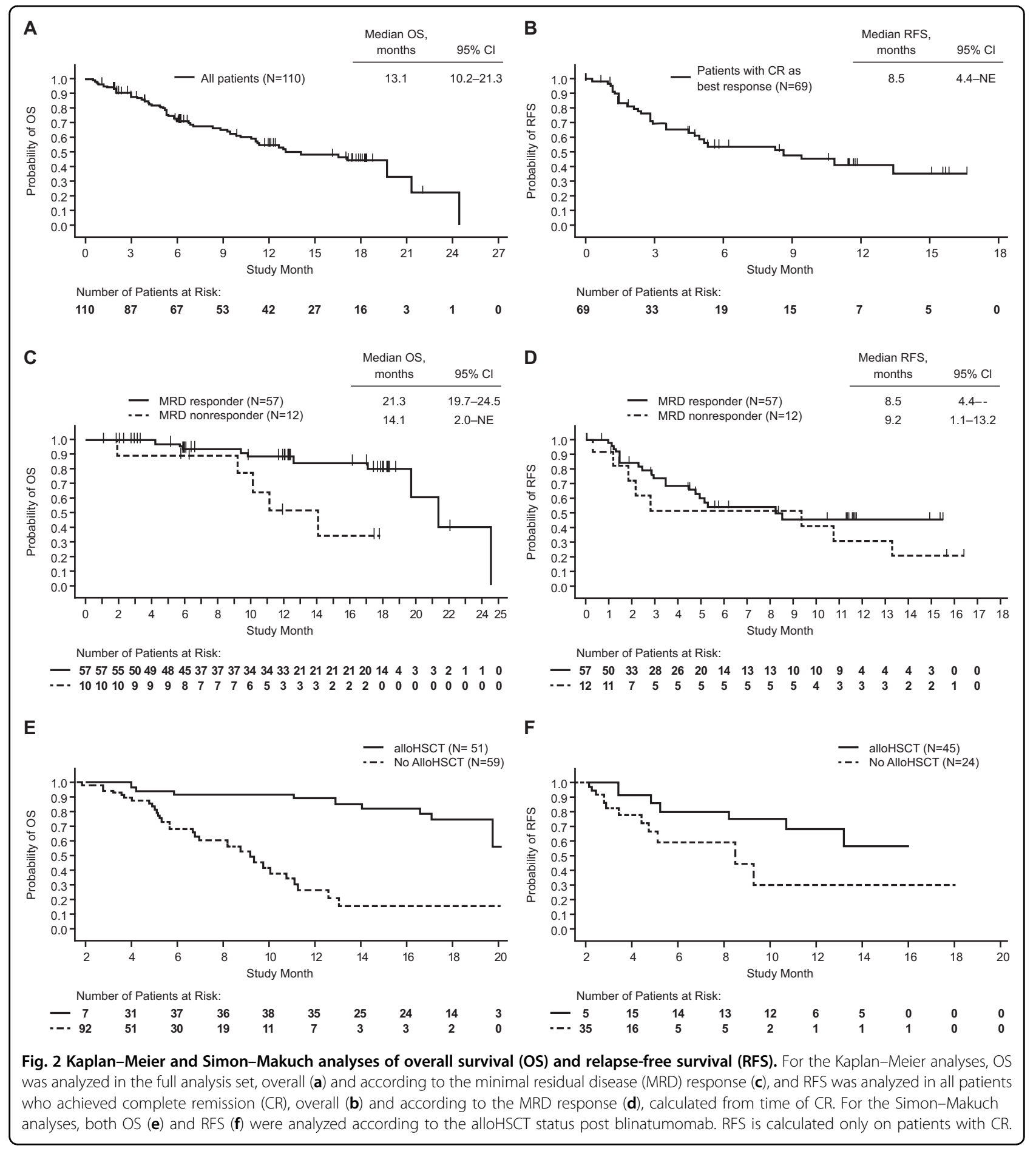

patients who received alloHSCT after blinatumomab as compared with those who did not (Fig. 2e, f). The Kaplan-Meier estimate of 100-day mortality following alloHSCT for these patients $(n=45)$ was $4.5 \%$ (95\% CI 1.2-17.0\%). Six of twenty-three relapses (26\%) were CD19-negative.
Median OS for all patients $(n=110)$ was 13.1 months (95\% CI 10.2-21.3), with a median follow-up of 17.4 months (Fig. 2a). For all patients reaching or maintaining CR in the first two cycles of blinatumomab $(n=$ 69), median RFS was 8.5 months (95\% CI 4.4-not evaluable), with a median follow-up of 11.2 months (Fig. 2b); 
23 patients (33\%) relapsed and $6(9 \%)$ died. Those who achieved MRD response had longer OS than those who achieved CR without MRD response; however, there was no apparent difference in RFS between these subgroups (Fig. 2c, d). For patients given HSCT in continuous CR, OS was nonestimable at the 15-month analysis for both the MRD responders and nonresponders groups (Supplementary Fig. 2).

Our findings demonstrate an acceptable safety profile and high response rate with blinatumomab in pediatric patients with $\mathrm{r} / \mathrm{r}$ BCP-ALL. Blinatumomab is generally better tolerated compared with chemotherapy ${ }^{5,8,9}$; however, it is associated with distinct AEs, including neurologic toxicities and CRS. In this study, such events at $\geq$ grade 3 were infrequent. Incidence of grade $3 / 4$ AEs was lower than in the recent phase 2 pediatric study ${ }^{7}$, possibly reflecting the lower tumor burden of patients recruited here. Overall, the safety profile was consistent with that reported in other studies of blinatumomab ${ }^{4,6,7}$.

Our efficacy findings showed a trend similar to that observed in the recent phase 2 study of blinatumomab in pediatric patients with $\mathrm{r} / \mathrm{r}$ BCP-ALL, in which a higher $\mathrm{CR}$ rate was seen in patients with lower baseline blast counts ${ }^{7}$. This explains the increased response rate observed here compared with the phase 2 trial, since a higher proportion of patients with $<50 \%$ blasts were enrolled $(61 \%$ of patients versus $26 \%$ in the phase 2 study) ${ }^{7}$.

Blinatumomab demonstrated a $78 \%$ molecular response rate in adult patients in morphological remission $(<5 \%$ blasts with MRD levels $\geq 10^{-3}$ ); ${ }^{4}$ ours is the first pediatric study to include patients with these disease characteristics. Of the 12 patients recruited, 11 achieved MRD response within the first two blinatumomab cycles, suggesting that further investigation in this patient population is warranted.

There was no apparent difference in response rates according to the number of prior relapses or prior alloHSCT. This contrasts with chemotherapy outcomes, where response rates decline with each consecutive relapse ${ }^{10}$. Only five patients had received prior blinatumomab, but four of five achieved CR, and three achieved MRD response, indicating that reexposure to blinatumomab may be effective, provided that leukemia blasts are still CD19-positive; CD19-negative relapse, a tumor-escape mechanism, indicates a poor prognosis ${ }^{11}$. Achievement of CR in three of four patients with constitutional trisomy 21 suggests that blinatumomab could be particularly useful to treat these fragile patients at risk of experiencing severe toxicities when exposed to aggressive chemotherapy ${ }^{12}$. Remarkably, both patients with $t(17 ; 19)$ rearrangement obtained $C R$ with MRD response, this finding corroborating recent data on blinatumomab efficacy in this peculiar patient subset $^{13}$.
A recent meta-analysis reported a significant OS benefit for pediatric patients who achieved MRD negativity relative to those who did not ${ }^{3}$. Here, Kaplan-Meier analyses show improved OS for patients who achieved MRD response as compared with those who did not, with no difference in RFS, possibly because of the low number of patients in the MRD nonresponder group $(n=12)$. Among patients with $\mathrm{CR}$ as best response in the first two blinatumomab cycles, $65 \%$ proceeded to alloHSCT, and these patients appeared to have longer RFS and OS compared with patients who did not subsequently receive alloHSCT, a finding consistent with the phase 2 pediatric trial $^{7}$. Our study is limited by the lack of a comparator arm and the assessment of outcomes by investigators rather than by independent central review.

Targeted immunotherapies other than blinatumomab are being developed and have demonstrated activity in pediatric patients with $\mathrm{r} / \mathrm{r}$ BCP-ALL. These include inotuzumab ozogamicin, although not approved for pediatric patients ${ }^{14}$, and CD19-directed chimeric antigen receptor $\mathrm{T}$-cell therapies ${ }^{15-17}$. These trials highlight expansion of treatment options beyond standard of care chemotherapeutic approaches in patients with $\mathrm{r} / \mathrm{r}$ BCP-ALL.

This expanded access study with blinatumomab in pediatric patients with $\mathrm{r} / \mathrm{r}$ BCP-ALL confirms a tolerable safety profile and indicates a response rate higher than previously reported, further supporting the use of blinatumomab for treatment of children with BCP-ALL.

\section{Acknowledgements}

This study was funded by Amgen, Inc. Medical writing assistance was provided by Karen O'Leary, PhD, and Lesley Blogg, PhD, of Fishawack Communications, Inc., and was funded by Amgen Inc.

\section{Author details}

'Department of Hematology and Oncology, IRCCS Bambino Gesù Children's Hospital, Rome, Sapienza, University of Rome, Rome, Italy. ${ }^{2}$ Amgen Research (Munich) GmbH, Munich, Germany. ${ }^{3}$ Department for Children and Adolescents, University Hospital Frankfurt, Frankfurt, Germany. ${ }^{4}$ St Jude Children's Research Hospital, Memphis, TN, USA. ${ }^{5}$ niversity Children's Hospital Wuerzburg, Wuerzburg, Germany. ${ }^{6}$ Department of Pediatric Oncology, Children's Research Centre, University Children's Hospital Zurich, Zurich, Switzerland. 'Department of Hematology/Oncology, University Children's Hospital Tuebingen,

Tuebingen, Germany. ${ }^{8}$ Pediatric Hematology and Immunology Department, Robert Debre Hospital, APHP, Paris, France. ${ }^{9}$ Department of Pediatric Hematology and Oncology, University Children's Hospital Muenster, Muenster, Germany. ${ }^{10}$ Department of Pediatrics, Division of Oncology and Hematology, Charité Universitätsmedizin Berlin, Berlin, Germany

\section{Data availability}

Qualified researchers may request data from Amgen clinical studies. Complete details are available at the following: http://www.amgen.com/datasharing.

\section{Conflict of interest}

F.L. reports speakers' bureau and consulting fees from Amgen Inc. G.Z. is employed by, holds stock in, and has patents/royalties/other intellectual property with Amgen Inc. N.M. is employed by and holds stock in Amgen Inc P.B. declares speakers' bureau fees from Amgen Inc., Novartis, Celgene, Medac, and Servier, and institutional research grants from Riemser, Medac, and Neovii. C.R. reports honoraria and consulting fees from Amgen Inc. Celgene, Novartis, Genentech, and Roche and honoraria from Pfizer, BMS, and Roche. S.J., P.-G.S., 
J.-P.B., R.H., and B.B. report consulting fees from Amgen Inc., and C.C.-S. has nothing to disclose.

\section{Publisher's note}

Springer Nature remains neutral with regard to jurisdictional claims in published maps and institutional affiliations.

Supplementary Information accompanies this paper at (https://doi.org/ 10.1038/s41408-020-00342-x).

Received: 12 February 2020 Revised: 18 June 2020 Accepted: 25 June 2020 Published online: 24 July 2020

\section{References}

1. Hunger, S. P. \& Mullighan, C. G. Acute lymphoblastic leukemia in children. N. Engl. J. Med. 373, 1541-1552 (2015).

2. Blanco, E. et al. Non-relapse mortality in pediatric acute lymphoblastic leukemia: a systematic review and meta-analysis. Leuk. Lymphoma 53, 878-885 (2012).

3. Berry, D. A. et al. Association of minimal residual disease with clinical outcome in pediatric and adult acute lymphoblastic leukemia: a meta-analysis. JAMA Oncol. 3, e170580 (2017).

4. Gokbuget, N. et al. Blinatumomab for minimal residual disease in adults with B-cell precursor acute lymphoblastic leukemia. Blood 131, 1522-1531 (2018).

5. Kantarjian, $H$. et al. Blinatumomab versus chemotherapy for advanced acute lymphoblastic leukemia. N. Engl. J. Med. 376, 836-847 (2017).

6. Martinelli, G. et al. Complete hematologic and molecular response in adult patients with relapsed/refractory Philadelphia chromosome-positive b-precursor acute lymphoblastic leukemia following treatment with blinatumomab: results from a Phase II, single-arm, multicenter study. J. Clin. Oncol. 35 1795-1802 (2017).

7. von Stackelberg, A. et al. Phase I/Phase II study of blinatumomab in pediatric patients with relapsed/refractory acute lymphoblastic leukemia. J. Clin. Oncol. 34, 4381-4389 (2016).

8. Stein, A. S. et al. Exposure-adjusted adverse events comparing blinatumomab with chemotherapy in advanced acute lymphoblastic leukemia. Blood Adv. 2, 1522-1531 (2018).

9. Hathaway, L., Sen, J. M. \& Keng, M. Impact of blinatumomab on patient outcomes in relapsed/refractory acute lymphoblastic leukemia: evidence to date. Patient Relat. Outcome Meas. 9, 329-337 (2018)

10. Sun, W. et al. Outcome of children with multiply relapsed B-cell acute lymphoblastic leukemia: a therapeutic advances in childhood leukemia \& lymphoma study. Leukemia 32, 2316-2325 (2018).

11. Ruella, M. \& Maus, M. V. Catch me if you can: leukemia escape after CD19directed T cell immunotherapies. Comput. Struct. Biotechnol. J. 14, 357-362 (2016).

12. Buitenkamp, T. D. et al. Acute lymphoblastic leukemia in children with Down syndrome: a retrospective analysis from the Ponte di Legno study group. Blood 123, 70-77 (2014).

13. Mouttet, B. et al. Durable remissions in TCF3-HLF positive acute lymphoblastic leukemia with blinatumomab and stem cell transplantation. Haematologica 104, e244-e247 (2019).

14. Bhojwani, D. et al. Inotuzumab ozogamicin in pediatric patients with relapsed/ refractory acute lymphoblastic leukemia. Leukemia 33, 884-892 (2018).

15. Gardner, R. A. et al. Intent-to-treat leukemia remission by CD19 CAR T cells of defined formulation and dose in children and young adults. Blood $\mathbf{1 2 9}$ 3322-3331 (2017).

16. Lee, D. W. et al. T cells expressing CD19 chimeric antigen receptors for acute lymphoblastic leukaemia in children and young adults: a phase 1 doseescalation trial. Lancet 385, 517-528 (2015).

17. Maude, S. L. et al. Chimeric antigen receptor T cells for sustained remissions in leukemia. N. Engl. J. Med. 371, 1507-1517 (2014). 